Check for updates

Cite this: Chem. Commun., 2020, 56,1290

Received 5th November 2019, Accepted 19th December 2019

DOI: $10.1039 / \mathrm{c} 9 \mathrm{cc} 08628 \mathrm{e}$

rsc.li/chemcomm

\section{Transition metal-free cyclobutene rearrangement in fused naphthalen-1-ones: controlled access to functionalized quinones $\uparrow$}

\author{
Fernando Herrera, ${ }^{a}$ Amparo Luna, (D) a Israel Fernández (D) ${ }^{b}$ and \\ Pedro Almendros (iD) *c
}

The controlled synthesis of 1,4-naphthoquinones and tetraphene7,12-diones, which bear the ABCD-ring of landomycins, has been accomplished directly through oxidative rearrangement of common stable precursors, namely, previously non-isolable cyclobuta[a]naphthalen$4(2 H)$-ones.

The ubiquity of the quinone moiety in natural products and organic materials justifies the continued interest in the synthesis of molecules containing this framework. ${ }^{1}$ The widespread occurrence of the cyclobutene nucleus in natural products and bioactive compounds, coupled to the use of this strained carbocycle as a building block in organic synthesis, triggered a renewed activity in the synthesis of cyclobutenes. $^{2}$ However, the preparation of the quinone core from cyclobutenes has remained unexplored. Jiang and co-workers have recently communicated the cyclization reactions of allenynones toward naphthols using DABSO [DABCO $\left.\left(\mathrm{SO}_{2}\right)_{2}\right]$ and arenediazonium salts (Scheme 1a), ${ }^{3}$ or either in presence of alkynes (Scheme 1b), ${ }^{4}$ or $\beta$-ketonitriles (Scheme 1c), ${ }^{5}$ involving the generation of tricyclic cyclobutene intermediates. Aiming to extend the utility of allenynones, we planned to use allenyne precursors bearing substituents at the internal allene double bond. Worthy of note, the presence of the extra-substituent $\left(\mathrm{R}^{3}=\mathrm{Me}, \mathrm{Ar}\right)$ did allow for the isolation of previously unstable and non-isolable tricyclic cyclobutenes $\left(\mathrm{R}^{3}=\mathrm{H}\right)$. Herein, we present a convenient method for the divergent synthesis of 1,4-naphthoquinones and tetraphene-7,12diones through the oxidative reorganization of cyclobutene-fused naphthalen-1-ones (Scheme 1d).

\footnotetext{
${ }^{a}$ Grupo de Lactamas y Heterociclos Bioactivos, Departamento de Quimica Orgánica, Unidad Asociada al CSIC, Facultad de Quimica, Universidad Complutense de Madrid, 28040-Madrid, Spain

${ }^{b}$ Departamento de Química Orgánica I and Centro de Innovación en Quimica Avanzada (ORFEO-CINQA), Facultad de CC. Quimicas, Universidad Complutense de Madrid, 28040-Madrid, Spain

' Instituto de Química Orgánica General, IQOG-CSIC, Juan de la Cierva 3, 28006-Madrid, Spain.E-mail: palmendros@iqog.csic.es

$\dagger$ In memory of Prof. Odón Arjona.

\# Electronic supplementary information (ESI) available: Experimental procedures, compound characterization data, and copies of NMR spectra for all new compounds. See DOI: 10.1039/c9cc08628e
}

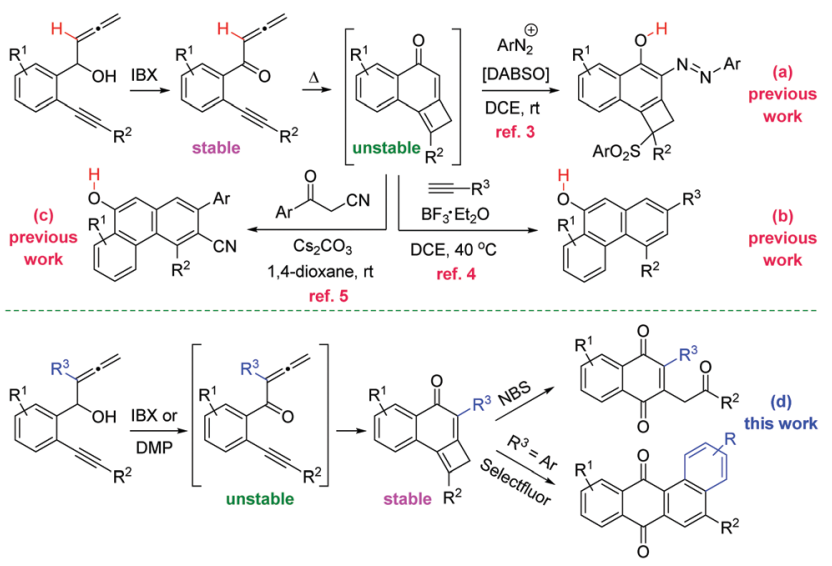

Scheme 1 The allenynone framework as a platform for the synthesis of fused carbocycles: prior art and our method.

Starting substrates, allenynols $\mathbf{2 a - k}$, were prepared from alkynyl-benzaldehydes $\mathbf{1}$ and 3-substituted prop-2-ynyl bromides using an indium-mediated allenylation under Barbier conditions in aqueous media (Scheme 2). Further oxidation with IBX or DessMartin periodinane (DMP) to introduce the carbonyl moiety gives rise directly to cyclobutene-fused naphthalen-1-ones $3 \mathbf{a}-\mathbf{k}$ (Scheme 2). It was found that the best results were systematically obtained in the presence of DMP. Because of the great reactivity imparted by the highly conjugated system in the initially formed non-isolable allenynones, [2+2] cycloaddition reactions take place spontaneously. ${ }^{6}$ Tricyclic cyclobutenes 3 were obtained with complete chemo- and regioselectivity in good yields.

To explore the reactivity of cyclobutene-fused naphthalen-1ones 3 , at the beginning of the study we selected $\mathrm{N}$-bromosuccinimide (NBS) as a convenient electrophilic source of bromine and tricyclic cyclobutene $\mathbf{3 a}$ as a model substrate. In the event, the use of THF as solvent led to reaction failure. Fortunately, the replacement of THF by acetonitrile provided 2-methyl-3-(2-oxo-2-phenylethyl)naphthalene-1,4-dione $4 a,{ }^{7}$ but only in a modest $29 \%$ yield. Because of the structure of $4 \mathbf{a}$, it may be apparent that adventitious water in the reaction 

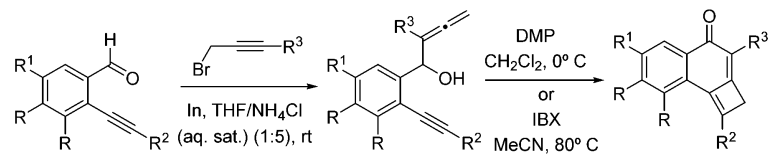

1a $R=H, R^{1}=H, R^{2}=P h$

1a $R=H, R^{1}=H, R^{2}=P h$

1b $R=H, R^{1}=H, R^{2}=T M S$

1c $R=H, R^{1}=H, R^{2}=4-M_{e O C} H_{4}$

1d $\mathrm{R}=\mathrm{H}, \mathrm{R}^{1}=\mathrm{H}, \mathrm{R}^{2}=4-\mathrm{MeC}_{6} \mathrm{H}_{4}$

1e $\mathrm{R}=\mathrm{H}, \mathrm{R}^{1}=\mathrm{H}, \mathrm{R}^{2}=4-\mathrm{BrC}_{6} \mathrm{H}_{4}$

1f $\mathrm{R}=\mathrm{H}, \mathrm{R}^{1}=\mathrm{H}, \mathrm{R}^{2}=2-\mathrm{MeC}_{6} \mathrm{H}_{4}$

$\lg R=H, R^{1}=F, R^{2}=P h$

1h $\mathrm{R}=\mathrm{H}, \mathrm{R}^{1}=\mathrm{MeO}, \mathrm{R}^{2}=\mathrm{Ph}$

1i $R=M e O, R^{1}=H, R^{2}=P h$

1i $R=M e O, R^{1}=H, R^{2}=P h$

2a $R^{3}=\mathrm{Me}(69 \%)$

2b R $\mathrm{R}^{3}=\mathrm{Ph}(60 \%)$

2c $\mathrm{R}^{3}=\mathrm{Ph}(65 \%)$

2d $R^{3}=P h(53 \%)$

2e $\mathrm{R}^{3}=\mathrm{Ph}(67 \%)^{\mathrm{a}}$

2f $\mathrm{R}^{3}=\mathrm{Ph}(49 \%)$

$2 \mathrm{~g} \mathrm{R}^{3}=\mathrm{Ph}(41 \%)^{\mathrm{a}}$

$2 h \mathrm{R}^{3}=\mathrm{Ph}(35 \%)^{\mathrm{a}}$

2i $\mathrm{R}^{3}=\mathrm{Ph}(68 \%)$

2j $R^{3}=\operatorname{Me}(77 \%)$

2k R $\mathrm{R}^{3}=\mathrm{Ph}(49 \%)$ 3a $(69 \%$ or $35 \%)$

3b $(82 \%$ or $65 \%)$

3c $(85 \%$ or $65 \%)$

3d $(88 \%$ or $51 \%)$

3e $(77 \%$ or $69 \%)$

3f $(80 \%$ or $58 \%)$

$3 g(82 \%$ or $45 \%)$

3h $(80 \%$ or $47 \%)$

$3 \mathbf{i}(70 \%$ or $52 \%)$

$3 \mathbf{j}(56 \%$ or $39 \%)$

3k $(81 \%$ or $57 \%$ )

Scheme 2 Synthesis of tricyclic cyclobutenes $3 \mathbf{a}-\mathbf{k} .{ }^{a}$ The reaction was carried out in $\mathrm{THF} / \mathrm{H}_{2} \mathrm{O}(1: 1)$.

medium was necessary. To prove this assumption, we carried out the reaction with the addition of 5.0 equiv. of water. Indeed, in this way a significant improvement of the yield of $4 \mathbf{a}$ up to $72 \%$ was achieved (Scheme 3 ). With the optimal conditions in hand, we evaluated the influence of substituents at the different positions of tricycles 3 . A variety of aromatic moieties were well tolerated both at $\mathrm{R}^{2}$ and $\mathrm{R}^{3}$, while an aliphatic substituent was accommodated at $\mathrm{R}^{3}$ (Scheme 3 ). Even precursor $\mathbf{3 j}$ bearing two electron-donating substituents $(\mathrm{R}=\mathrm{MeO})$ on the aromatic ring, conveniently afforded naphthalene-1,4-dione $\mathbf{4 j}$ after NBS treatment. By contrast, the reaction of TMS-cyclobutene $2 \mathrm{c}$ was not satisfactory. Interestingly, the presence of a fluorine atom at the benzene ring such as in tricyclic cyclobutene $\mathbf{3 h}$ resulted in an extra-bromination on the final adduct $\mathbf{4 h}-\mathbf{B r}$ (Scheme 3).

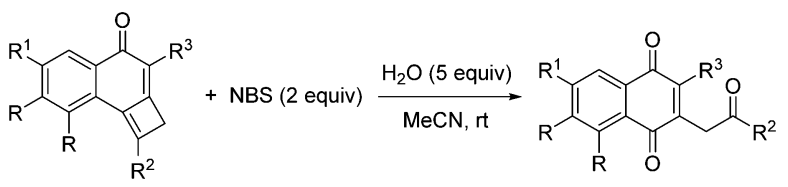

$\begin{array}{ll}\text { 3a } R=H, R^{1}=H, R^{2}=P h, R^{3}=M e & \text { 4a }(72 \%) \\ \text { 3b } R=H, R^{1}=H, R^{2}=P h, R^{3}=P h & \text { 4b (61\%) } \\ \text { 3c } R=H, R^{1}=H, R^{2}=T M S, R^{3}=P h & \text { 4c }(0 \%) \\ \text { 3d } R=H, R^{1}=H, R^{2}=4-M e C_{6} H_{4}, R^{3}=P h & \text { 4d }(58 \%) \\ \text { 3e } R=H, R^{1}=H, R^{2}=4-M_{6} C_{6} H_{4}, R^{3}=P h & 4 e(55 \%) \\ \text { 3f } R=H, R^{1}=H, R^{2}=4-B_{6} H_{4}, R^{3}=P h & 4 f(60 \%) \\ \text { 3i } R=H, R^{1}=M e O, R^{2}=P h, R^{3}=P h & 4 i(53 \%) \\ \text { 3j } R=M e O, R^{1}=H, R^{2}=P h, R^{3}=M e & \text { 4j (57\%) }\end{array}$

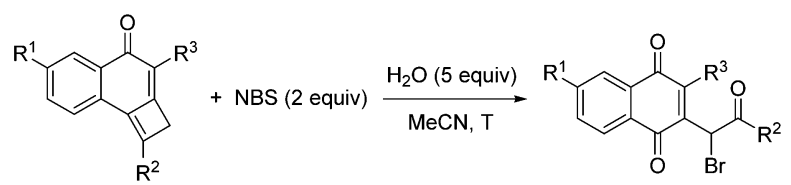

3a $R^{1}=H, R^{2}=P h, R^{3}=M e$

3h $R^{1}=F, R^{2}=P h, R^{3}=P h$

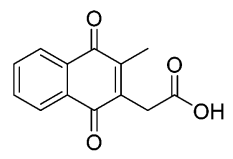

inhibitor of glutathione reductase
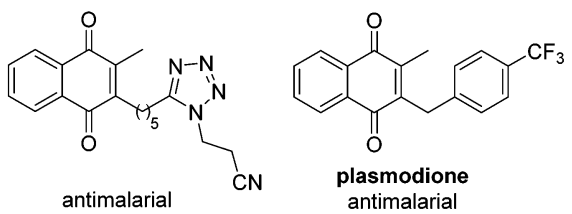

Scheme 3 Synthesis of 2-substituted-3-(2-oxo-2-arylethyl)naphthalene1,4-diones 4 and $\mathbf{4 - B r}$.
The same phenomenon was observed starting from tricycle 3a under otherwise identical reaction conditions but through mild heating, which resulted in the formation of $\mathbf{4 a - B r}$ (Scheme 3). Pleasingly, it has been reported that several 2-methyl-3-alkylnaphthalene-1,4-diones related to bicycles 4 possess interesting biological activities. ${ }^{8}$

A plausible mechanism for the NBS-promoted genesis of 1,4-naphthoquinones 4 is delineated in Scheme 4. The reaction is presumed to possess a radical nature, which was based on the suspension of the transformation after the addition of TEMPO to the reaction medium. Initially, the formation of allylic radical species INT-1 should occur by bromine radical attack. The formation of this radical should be followed by oxidation to the carbocationic species INT-2 by single electron transfer (SET) to the succinimide. Next, water attack takes place with formation of bromohydrin INT-3 which is followed by $\mathrm{HBr}$ release. Hydration of the resulting intermediate cyclobutenol INT-4 leads to diol INT-5, which evolves into zwitterionic intermediate INT-6. According to Density Functional Theory calculations (see ESI $\%$ ), this step proceeds with a low activation barrier of only $10.6 \mathrm{kcal} \mathrm{mol}^{-1}$ (for 3a). A subsequent proton transfer leads to dihydroquinone $\mathbf{4 H}$ in a highly exergonic transformation $\left(\Delta G_{\mathrm{R}}=-40.3 \mathrm{kcal} \mathrm{mol}^{-1}\right.$, for $\left.3 \mathrm{a}\right)$, which is finally oxidized to its quinone form 4 .

Aiming to explore the effect of different reagents in the selective oxidative reorganization of cyclobutene-fused naphthalen-1-ones, we decided to expose tricycles 3 to the action of Selectfluor. The reaction of adduct $\mathbf{3 a}$ with Selectfluor was problematic and a complex mixture was obtained. By contrast, an encouraging result was obtained when adduct $\mathbf{3 b}$ having a phenyl group was used, because we unexpectedly isolated in a $19 \%$ yield tetracycle $\mathbf{5 b}$ that should arise from an angular benzannulation process. The presence of sodium bicarbonate in the reaction between Selectfluor and $\mathbf{3 b}$ caused an appreciable rise in the yield of $\mathbf{5 b}$ (Scheme 5). On this point, we next tested the scope of this rearrangement reaction using diverse fused-cyclobutenes 3 which contain a phenyl moiety at the cyclohexenone ring. A variety of differently substituted precursors 3, including fluoroand methoxy-derivatives $\mathbf{3 h}$ and $\mathbf{3 i}$ were suitably rearranged. By contrast, the reaction of dimethoxy-substituted precursor $3 \mathbf{k}$ with Selectfluor was troublesome and the desired tetracycle 5k was not isolated in reasonable purity. Synthetically useful yields of tetracycles $\mathbf{5 b}$ and $\mathbf{5 d - i}$ were attained (Scheme 5). For all examples included in Scheme 5 no products of type 4 were observed.

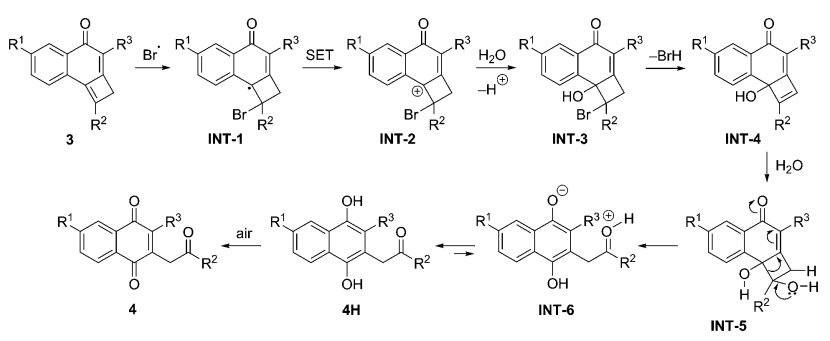

Scheme 4 Mechanistic explanation for the NBS-promoted synthesis of 2-oxo-naphthalene-1,4-diones 4. 


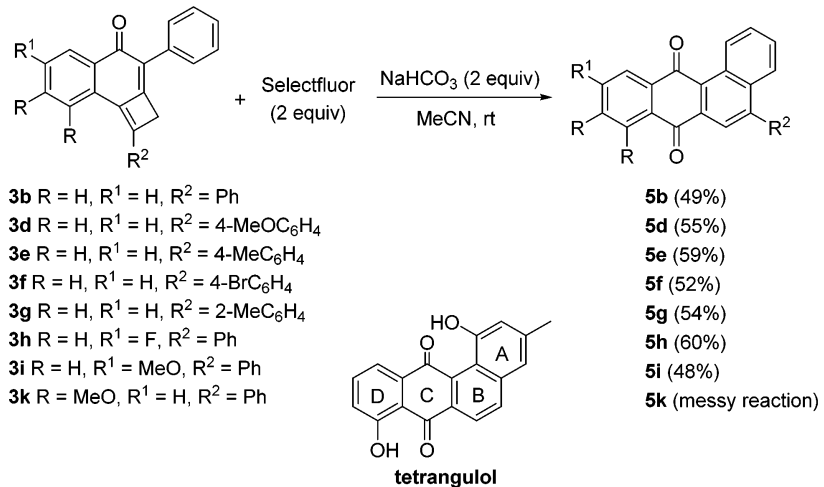

Scheme 5 Synthesis of 5-aryltetraphene-7,12-diones $\mathbf{5 b}$ and $\mathbf{5 d - i}$.

Noteworthy, tetracycles 5 bear the ABCD-ring of landomycins. Landomycins such as tetrangulol are a class of natural quinones characterized by an angular conjugated tetracyclic core, which confers them with interesting bioactivities. ${ }^{9}$ The major difficulty associated with the synthesis of landomycins is the formation of the B-ring due to hindrance issues. This elusive aspect is easily addressed in our preparation of the landomycin core.

In order to gain some mechanistic insights, control experiments were planned (Scheme 6). First, when tricycle $\mathbf{3 b}$ was treated with Selectfluor under the optimized conditions but using anhydrous acetonitrile, the yield of $\mathbf{5 b}$ decreased to $8 \%$ (Scheme 6). This result unveiled the origin of the oxygen at the C-ring, that should come from ambient water. Succeeding in performing a divergent preparation of 2-substituted-3-(2-oxo2-arylethyl)naphthalene-1,4-diones 4 and 5-aryltetraphene-7,12diones 5 , we speculated about the possible intermediacy of 1,4-naphthoquinones 4 in the formation of angular tetracycles $\mathbf{5}$ in the presence of Selectfluor. When 1,4-naphthoquinone $\mathbf{4 b}$ was treated with Selectfluor and sodium bicarbonate under the optimized reaction conditions for the formation of pentacycles $\mathbf{5}$, the reaction failed and starting material $\mathbf{4 b}$ was fully recovered (Scheme 6). From the above experiment, it may be inferred that naphthalene-1,4-diones of type $\mathbf{4}$ should be discarded as intermediates for this reaction. The radical scavenger TEMPO effectively suppressed the formation of the required product, pointing to a radical reaction mechanism.
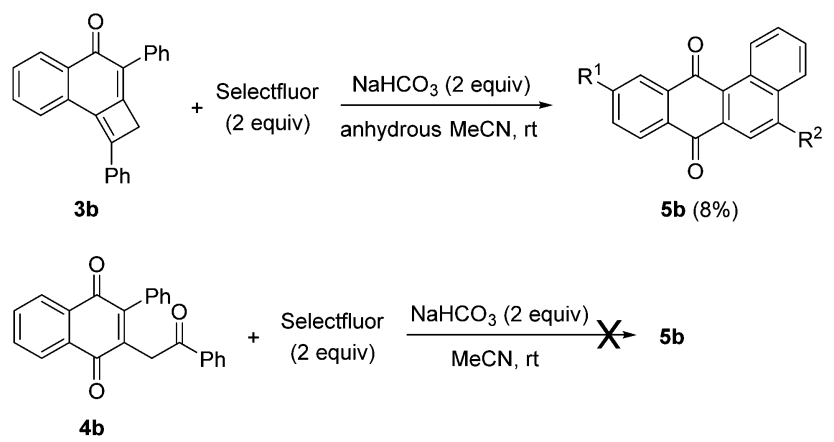

3b $\frac{\text { optimal conditions }}{\text { TEMPO }} \times \mathbf{5 b}$

Scheme 6 Control experiments.

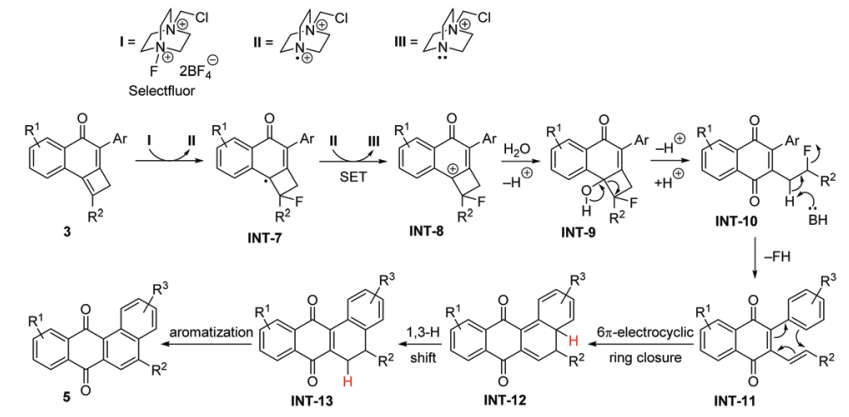

Scheme 7 Mechanistic explanation for the Selectfluor-promoted synthesis of tetraphene-7,12-diones $\mathbf{5}$.

Based on the above experiments, we assume a mechanism as presented in Scheme 7 for the reaction of aryl-substituted cyclobutene-fused naphthalen-1-ones 3 with Selectfluor. Similar to the above-described reaction involving NBS, tricycles 3 can be converted into cationic intermediates INT-8 after Selectfluor treatment with concomitant formation of III. Next, this intermediate should suffer water attack to produce halohydrin INT-9, which evolves through cyclobutane ring opening followed by baseassisted HF release ${ }^{10}$ into the quinone INT-11. The so-formed polyene is able to undergo a $6 \pi$-electrocyclic ring closure to produce INT-12, ${ }^{11}$ which rapidly evolves into INT-13 via a 1,3-hydrogen migration. According to DFT calculations on $\mathbf{3 b}$ (see ESI $\neq$ ), this step proceeds with an activation barrier of $21.9 \mathrm{kcal} \mathrm{mol}^{-1}$ in a highly exergonic transformation $\left(\Delta G_{\mathrm{R}}=-28.2 \mathrm{kcal} \mathrm{mol}^{-1}\right)$ driven by the gain in aromaticity in the system. Finally, INT-13 is oxidized into the observed tetracycles 5 . Our calculations indicate that the concerted $\mathrm{H}_{2}$ release from either INT-13 or even from INT-12 thus directly forming 5 is unfeasible $\left(\Delta G^{\ddagger}=89.9\right.$ and $77.4 \mathrm{kcal} \mathrm{mol}^{-1}$, respectively). This points to a different mechanism for this final aromatization step, ${ }^{12}$ which is not evident to us at the moment.

In conclusion, we have developed a divergent outcome transformation of the previously non-isolable cyclobuta $[a]$ naphthalen$4(2 \mathrm{H})$-one system to afford either 1,4-naphthoquinones or tetraphene-7,12-diones, which has been accomplished through the reorganization of the above fused tricyclic cyclobutenes in the presence of NBS or Selectfluor.

This work was supported in part by AEI (MICIU) and FEDER (Project PGC2018-095025-B-I00 to P. A. and CTQ2016-78205-P and CTQ2016-81797-REDC to I. F.). F. H. thanks UCM for a predoctoral contract. We are grateful to $\mathrm{M}$. Tiemblo in our group for the preparation of compounds $2 \mathbf{j}$, $2 \mathbf{k}$ and $3 \mathbf{j}$ and Prof. B. Alcaide for continued support.

\section{Conflicts of interest}

There are no conflicts of interest to declare.

\section{Notes and references}

1 (a) S. Liu, T. Shen, Z. Luo and Z.-Q. Liu, Chem. Commun., 2019, 55, 4027 and references therein. For representative reviews, see: (b) Y. Wang, S. Zhu and L.-H. Zou, Eur. J. Org. Chem., 2019, 2179; (c) Y. Ando and K. Suzuki, Chem. - Eur. J., 2018, 24, 15955; (d) E. J. Son, J. H. Kim, K. Kim and C. B. Park, J. Mater. Chem. A, 
2016, 4, 11179; (e) S. N. Sunassee and M. T. Davies-Coleman, Nat. Prod. Rep., 2012, 29, 513; $(f)$ C. Asche, Mini-Rev. Med. Chem., 2005, 5, 449; $(g)$ R. H. Thomson, Naturally Occurring Quinones IV, Blackie Academic \& Professional, London, 1997; (h) P. J. O'Brien, Chem.-Biol. Interact., 1991, 80, 1; (i) The Chemistry of the Quinonoid Compounds, ed. S. Patai and Z. Rappoport, Wiley, New York, 1988.

2 For recent references, see: (a) Q. Qin, X. Luo, J. Wei, Y. Zhu, X. Wen, S. Song and N. Jiao, Angew. Chem., Int. Ed., 2019, 58, 4376; (b) G.-Q. Xu, J.-T. Xu, Z.-T. Feng, H. Liang, Z.-Y. Wang, Y. Qin and P.-F. Xu, Angew. Chem., Int. Ed., 2018, 57, 5110; (c) T. Iwai, M. Ueno, H. Okochi and M. Sawamura, $A d v$. Synth. Catal., 2018, 360, 670; (d) M. E. de Orbe, L. Amenós, M. S. Kirillova, Y. Wang, V. López-Carrillo, F. Maseras and A. M. Echavarren, J. Am. Chem. Soc., 2017, 139, 10302; (e) M. Eisold, A. N. Baumann, G. M. Kiefl, S. T. Emmerling and D. Didier, Chem. - Eur. J., 2017, 23, 1634; $(f)$ B. Alcaide, P. Almendros and C. Lázaro-Milla, Chem. - Eur. J., 2016, 22, 8998; ( $g$ ) Y. Qiu, B. Yang, C. Zhu and J.-E. Bäckvall, Angew. Chem., Int. Ed., 2016, 55, 6520; (h) B. Alcaide, P. Almendros, I. Fernández and C. Lázaro-Milla, Chem. Commun., 2015, 51, 3395.

3 (a) F. Liu, J.-Y. Wang, P. Zhou, G. Li, W.-J. Hao, S.-J. Tu and B. Jiang, Angew. Chem., Int. Ed., 2017, 56, 15570. Fan has described the preparation of cyclobutanol-fused 2-nitro-naphthalen-1-ols: $(b)$ T. Feng, Y. He, X. Zhang and X. Fan, Adv. Synth. Catal., 2019, 361, 1271.

4 (a) H. Li, W.-J. Hao, M. Wang, X. Qin, S.-J. Tu, P. Zhou, G. Li, J. Wang and B. Jiang, Org. Lett., 2018, 57, 4362; (b) R. Fu, M.-F. Li, P. Zhou, W.-J. Hao, S.-J. Tu and B. Jiang, Adv. Synth. Catal., 2019, 361, 2280; (c) B.-Z. Tang, J.-Z. Li, A.-W. Zhang, W.-J. Hao, S.-J. Tu and B. Jiang, Adv. Synth. Catal., 2019, 361, 3394.

5 (a) H.-K. Sha, T. Xu, F. Liu, B.-Z. Tang, W.-J. Hao, S.-J. Tu and B. Jiang, Chem. Commun., 2018, 54, 10415; (b) J.-Y. Wang, F.-L. Xie, J.-Q. Hu, S.-Z. Yang, Y.-J. Wang, W.-J. Hao, S.-J. Tu and B. Jiang, Org. Biomol. Chem., 2018, 16, 7104.

6 For a review on $[2+2]$ cycloaddition chemistry with allenes, see: B. Alcaide, P. Almendros and C. Aragoncillo, Chem. Soc. Rev., 2010, 39, 783.

7 Naphthoquinones have attracted considerable interest because of their relevant chemical and biological properties: $(a) \mathrm{K}$. W. Wellington, $R S C$ Adv., 2015, 5, 20309; (b) R. L. de Carvalho, G. A. M. Jardim, A. C. C. Santos, M. H. Araujo, W. X. C. Oliveira, A. C. S. Bombaça, R. F. S. Menna-Barreto, E. Gopi, E. Gravel, E. Doris and E. N. da Silva, Jr.,
Chem. - Eur. J., 2018, 24, 15227 and references therein; (c) A. S. Kumar, G. Thirupathi, G. S. Reddy and D. B. Ramachary, Chem. - Eur. J., 2019, 25, 1177; (d) S. Peraka, M. A. Pasha, G. Thirupathi and D. B. Ramachary, Chem. - Eur. J., 2019, 25, 14036.

8 (a) C. Biot, H. Bauer, R. H. Schirmer and E. Davioud-Charvet, J. Med. Chem., 2004, 47, 5972; (b) E. Davioud-Charvet, S. Delarue, C. Biot, B. Schwöbel, C. C. Böhme, A. Müssigbrodt, L. Maes, C. Sergheraert, P. Grellier, R. H. Schirmer and K. Becker, J. Med. Chem., 2001, 44, 4268; (c) L. Feng, D. A. Lanfranchi, L. Cotos, E. Cesar-Rodo, K. Ehrhardt, A. A. Goetz, H. Zimmermann, F. Fenaille, S. A. Blandin and E. Davioud-Charvet, Org. Biomol. Chem., 2018, 16, 2647.

9 C.-J. Sie, V. Patteti, Y.-R. Yang and K.-K. T. Mong, Chem. Commun., 2018, 54, 1885 and references therein.

10 (a) L. Xu, Q. Zhang, Q. Xie, B. Huang, J.-J. Dai, J. Xu and H.-J. Xu, Chem. Commun., 2018, 54, 4406; (b) C. Gerleve, M. Kischkewitz and A. Studer, Angew. Chem., Int. Ed., 2018, 57, 2441; (c) X.-Q. Chu, B.-Q. Cheng, Y.-W. Zhang, D. Ge, Z.-L. Shen and T.-P. Loh, Chem. Commun., 2018, 54, 2615; (d) S.-L. Zhang and J.-J. Dong, Org. Lett., 2019, 21, 6893; (e) C. Li, J.-M. Yuan, W. Chen, Y. He, J. Huang, Y. Huang, Q. Xiao, J. Sheng and C. Huang, Chem. - Asian J., 2019, 14, 2584; $(f)$ J. Qian, J. Zhang, H. Yang, L. Kang and G. Jiang, Chem. Sci., 2019, 10, 8812; $(g)$ Y. Chen, L. Li, X. He and Z. Li, ACS Catal., 2019, 9, 9098.

11 For recent use of $6 \pi$-electrocyclization in the synthesis of polycycles, see: (a) H. Kim, Y. J. Hwang, I. Han and J. M. Joo, Chem. Commun., 2018, 54, 6879; (b) B. Alcaide, P. Almendros, C. Lázaro-Milla and P. Delgado-Martínez, Chem. - Eur. J., 2018, 24, 8186; (c) M. P. Ball-Jones, J. Tyler, H. Mora-Radó, W. Czechtizky, M. Méndez and J. P. A. Harrity, Org. Lett., 2019, 21, 6821; (d) C. Elgindy, J. S. Ward and M. S. Sherburn, Angew. Chem., Int. Ed., 2019, 58, 14573.

12 For recent mechanistic proposals involving the conversion of intermediate dihydroarenes into the corresponding arenes which is associated with an oxidation step, probably due to the presence of atmospheric oxygen, see: (a) A. J. Ansari, A. A. Wani, A. K. Maurya, S. Verma, V. K. Agnihotri, A. Sharon, P. V. Bharatam and D. M. Sawant, Chem. Commun., 2019, 55, 14825; (b) G. Bianchini, P. Ribelles, D. Becerra, M. T. Ramos and J. C. Menéndez, Org. Chem. Front., 2016, 3, 412; (c) Q. Xu, P. Gu, F. Wang and M. Shi, Org. Chem. Front., 2015, 2, 1475. 